\title{
Non-Functioning Corticotroph Adenoma
}

National Cancer Institute

\section{Source}

National Cancer Institute. Non-Functioning Corticotroph Adenoma. NCI Thesaurus. Code C154429.

A pituitary gland corticotroph adenoma not associated with a hormonal syndrome. 